\section{Diagnóstico da tuberculose: desempenho do primeiro serviço de saúde procurado em São José do Rio Preto, São Paulo, Brasil}

\section{Tuberculosis diagnosis and performance assessment of the first health service used by patients in São José do Rio Preto, São Paulo State, Brazil}

\author{
Diagnóstico de la tuberculosis: desempeño del \\ primer servicio de salud solicitado en São José \\ do Rio Preto, São Paulo, Brasil
}

Maria Amélia Zanon Ponce 1 Anneliese Domingues Wysocki 1 Beatriz Estuque Scatolin 1 Rubia Laine de Paula Andrade 1 Tiemi Arakawa 1 Antonio Ruffino Netto 2 Aline Aparecida Monroe 2 Lucia Marina Scatena 3 Sílvia Helena Figueiredo Vendramini 4 Tereza Cristina Scatena Villa ${ }^{1}$

\title{
Resumo
}

${ }^{1}$ Escola de Enfermagem de Ribeirão Preto, Universidade de São Paulo, Ribeirão Preto, Brasil.

2 Faculdade de Medicina de Ribeirão Preto, Universidade de São Paulo, Ribeirão Preto Brasil.

3 Departamento de Medicina Social, Universidade Federal do Triangulo Mineiro, Uberaba, Brasil. ${ }^{4}$ Departamento de Enfermagem em Saúde Coletiva e Orientação Profissional, Faculdade de Medicina de São José do Rio Preto, São José do Rio Preto,

Correspondência M. A. Z. Ponce Escola de Enfermagem de Ribeirão Preto, Universidade de São Paulo Av. Bandeirantes 3900, Ribeirão Preto, SP 14040-902, Brasil. amelinha_famerp@yahoo. com.br

\begin{abstract}
This cross-sectional study aimed to analyze the first health service to which patients turned for tuberculosis diagnosis in São José do Rio Preto, São Paulo, Brazil, 2009. Eighty-one patients in treatment were interviewed with a questionnaire based on the Primary Care Assessment Tool adapted to TB care and used as a reference for health services performance (structure and process). Data analysis used descriptive and multiple correspondence techniques. Nearly half of the patients initially came to emergency care departments (49.4\%), and most TB diagnoses were made in hospitals (39.5\%) and primary care services (30.9\%). Specialized services were associated with the best diagnostic performance, as opposed to emergency departments, with the worst performance. Primary care services were associated with intermediate performance and weaknesses in infrastructure. The reach of effective detection of TB cases in the portals of entry into the health system involves enhanced diagnostic suspicion, thus emphasizing the need for investments in the management of human resources training for the identification of individuals with respiratory symptoms.
\end{abstract}

Tuberculosis; Delayed Diagnosis; Health Services; Primary Health Care
Estudo transversal com objetivo de analisar o desempenho do primeiro serviço de saúde procurado para o diagnóstico da TB pulmonar em São José do Rio Preto, São Paulo, Brasil, em 2009. Foram entrevistados 81 doentes em tratamento. Adotou-se um questionário baseado no Primary Care Assessment Tool, adaptado para atenção à $T B$, e o referencial de avaliação dos serviços de saúde (estrutura e processo). Os dados foram analisados mediante técnicas descritivas e análise de correspondência múltipla. O principal serviço procurado foi o Pronto Atendimento (UPA) $(49,4 \%)$ e o que mais diagnosticou os casos de TB foi o hospital (39,5\%) e a atenção básica (30,9\%). Os serviços especializados apresentaram associação com o melhor desempenho no diagnóstico e a UPA com o pior. A atenção básica associou-se com desempenho intermediário e fragilidades na estrutura. O alcance de uma detecção eficaz dos casos de TB nas principais portas de entrada perpassa pela melhoria na suspeição, reforçando a necessidade de investimentos da gestão na capacitação dos recursos humanos para a identificação dos sintomáticos respiratórios.

Tuberculose; Diagnóstico Tardio; Serviços de Saúde; Atenção Primária à Saúde 


\section{Introdução}

Os sistemas de saúde, em geral, organizam-se com base em uma porta de entrada que corresponde ao primeiro serviço de saúde procurado pelo usuário, a cada novo problema ou necessidade 1. Essa entrada deve ser de fácil acesso e dispensar atenção adequada e resolutiva, garantindo, quando necessário, a continuidade da assistência em outros serviços que integram as redes de atenção à saúde 2 . Neste sentido, o acesso universal, igualitário e ordenado às ações e serviços de saúde se inicia pelas portas de entrada do Sistema Único de Saúde (SUS) e se completa na rede regionalizada e hierarquizada, de acordo com a complexidade do serviço.

No processo de consolidação do SUS, a atenção básica é concebida como a porta de entrada preferencial e o eixo estruturante do sistema de saúde ${ }^{3}$, devendo assumir três funções essenciais: resolubilidade (capacidade cognitiva e tecnológica para solucionar mais de $85 \%$ das necessidades de saúde), coordenação (ordenar fluxos e contrafluxos de pessoas, produtos e informações entre os diferentes níveis de atenção, atuando como coordenador do cuidado) e a responsabilização pela população adscrita (por meio do conhecimento do contexto cultural, epidemiológico, econômico e social, pode-se estabelecer uma gestão fundamentada nas necessidades de saúde da comunidade) 2,4,5.

Além da atenção básica, recentemente, o Ministério da Saúde reconheceu como porta de entrada os serviços de atendimento inicial à saúde do usuário no SUS e habilitou outras portas de entrada para o sistema de saúde 6 , dentre elas, os serviços de atenção psicossocial, os serviços especiais de acesso aberto (como os ambulatórios de referência em HIV/AIDS e tuberculose) e as Unidades de Pronto Atendimento (UPA), que se voltam ao atendimento imediato e provisório de casos agudos ou de agudização de condições crônicas, sem necessidade de agendamento prévio, caracterizado pela atenção à queixa/problema/necessidade seguida de conduta/solução ?.

No entanto, apesar dos reconhecíveis avanços na tentativa de aproximar a organização do sistema de saúde, a real busca por serviços e ações de saúde, no Brasil, ainda há um descompasso entre o sistema de atenção (atendimento voltado às condições agudas e agudizações de condições crônicas) e a situação de saúde (caracterizada pela tripla carga de doença no país) 8 .

Nesse cenário, insere-se a TB, considerada uma condição crônica pela Organização Mundial da Saúde (OMS) 9, e que impõe, dentre muitos desafios, o diagnóstico oportuno dos casos, espe- cialmente nos serviços que atuam como porta de entrada do sistema de saúde 10,11,12,13.

Assim, o desempenho de tais serviços para o diagnóstico da doença é essencial para possibilitar a diminuição da morbi-mortalidade e a eliminação das fontes de infecção na comunidade 14 . Porém, na literatura ainda são escassos os estudos com essa perspectiva. Em um levantamento bibliográfico referente ao período de 2007 a 2011 nas bases de dados LILACS, MEDLINE e SciELO, dos 145 artigos publicados, apenas $10 \%$ abordavam o desempenho dos serviços e programas para o diagnóstico da TB $15,16,17,18,19,20$.

Destaca-se ainda que somente dois estudos foram realizados no Brasil, ambos com enfoque na avaliação dos serviços de atenção básica para o diagnóstico da TB, e mostraram que embora as Unidades de Saúde da Família (USF) pareçam facilitar o acesso à assistência próxima ao local de residência dos usuários, não foi verificada melhoria em relação ao diagnóstico oportuno e acompanhamento dos casos de TB 15,20.

Diante do exposto, este estudo teve como objetivo analisar o desempenho do primeiro serviço de saúde procurado (porta de entrada) para o diagnóstico da TB em São José do Rio Preto, São Paulo, Brasil.

\section{Material e métodos}

Estudo transversal realizado em São José do Rio Preto, município paulista de grande porte, prioritário para o controle da TB no país. Segundo o Instituto Brasileiro de Geografia e Estatística (IBGE), a população estimada para 2009 foi de 419.633 habitantes (IBGE. http://www.ibge.gov. br/censos, acessado em 24/Nov/2009), apresentando-se eminentemente urbana com uma densidade demográfica de 907,98 habitantes $/ \mathrm{km}^{2}$. No que concerne aos indicadores sociais, apresentou taxa de analfabetismo em torno de $3,2 \%$, índice de desenvolvimento humano de 0,834 e índice de Gini de 0,47.

Em 2009, a rede municipal de atenção à saúde se organizava de forma regionalizada em cinco distritos sanitários, composto por 13 Unidades Básicas de Saúde (UBS), 11 USF (cobertura populacional de 14,2\%), 5 UPA, 1 Ambulatório Regional de Especialidades (Núcleo de Gestão Ambulatorial - NGA-60) com Programa de Controle da Tuberculose (PCT), 1 ambulatório especializado em DST/AIDS (Serviço de Atendimento Especializado - SAE) e 6 hospitais.

$\mathrm{O}$ atendimento aos doentes de $\mathrm{TB}$ - incluindo diagnóstico e tratamento - é de responsabilidade das equipes generalistas da atenção básica do 
município com retaguarda da equipe especializada do PCT desde 2007.

Além do apoio, suporte técnico e treinamento para as unidades de atenção básica, a equipe do PCT é responsável pela gestão do programa, alimentação dos sistemas de informação da doença, além de oferecer o atendimento clínico aos casos mais complexos. A maior parte dos doentes de TB coinfectados pelo HIV/AIDS é acompanhada no serviço de atendimento especializado em doenças infectocontagiosas (SAE).

Em relação aos dados epidemiológicos da TB, o município apresentou um coeficiente de incidência de 23,1/100.000, sendo alarmante a alta taxa de coinfecção TB/HIV de 23\% em 2009. Neste mesmo ano, a taxa de detecção de casos da doença foi de $73,2 \%$. Em relação ao desfecho do tratamento, em 2009, a taxa de cura foi de $77,7 \%$, o abandono foi de $6,8 \%$ e o óbito foi de $15,5 \%$, estando ainda aquém dos parâmetros recomendáveis 21 .

A população de estudo foi constituída por doentes de TB pulmonar residentes no município, maiores de 18 anos, que realizaram tratamento no período de novembro de 2008 a novembro de 2009. Excluíram-se doentes pertencentes ao sistema prisional e que tiveram como desfechos mudança de diagnóstico, por não se constituir caso de TB, e transferência para outro município, uma vez que não foi possível localizar esses doentes. Após o levantamento do número de doentes nas bases de dados do sistema de informação TBWEB (Sistema Estadual de Notificação da Tuberculose. http://www.cvetb.saude.sp.gov. br), encontraram-se, no período supracitado, 119 doentes que realizaram tratamento para TB no município. Dentre eles, 92 doentes atendiam aos critérios de inclusão e 81 constituíram a população final do estudo, havendo 09 recusas, 01 exclusão por não saber informar o primeiro serviço de saúde procurado e 01 por estar internado e não ter condições de participar da pesquisa.

Os dados foram coletados no período de julho a dezembro de 2009 por meio de entrevistas com o doente de TB, utilizando um questionário estruturado elaborado com base no Primary Care Assessment Tool (PCAT) validado para avaliar os serviços de atenção primária no Brasil 22 e adaptado para atenção à TB 23 .

Para analisar o desempenho (grau de alcance dos objetivos dos sistemas de saúde 24) do primeiro serviço de saúde procurado (porta de entrada) para o diagnóstico da TB, foram utilizadas as seguintes variáveis: procura pelo serviço de saúde mais próximo do domicílio; obtenção de consulta em 24 horas, hipótese diagnóstica, solicitação de baciloscopia, solicitação de raio-x, encaminhamento para outro serviço de saúde, número de vezes que procurou o serviço de saúde até o diagnóstico, ocorrência de atraso no diagnóstico da TB e diagnóstico realizado no primeiro serviço de saúde procurado.

A variável "ocorrência de atraso no diagnóstico da TB" foi definida por um ponto de corte (valor mediano), pela informação fornecida pelos doentes sobre o tempo (dias) decorrido entre a procura pelo primeiro serviço de saúde ao início dos sintomas e o diagnóstico da TB. Logo, quando os doentes levaram $\geq 15$ dias, esse tempo foi avaliado como atraso. $\mathrm{O}$ uso da mediana foi mais adequado, uma vez que a distribuição dos valores referentes à variável "tempo" é assimétrica, amenizando a interferência de valores aberrantes.

Os dados foram analisados a partir de técnicas descritivas e análise fatorial de correspondência múltipla. A análise fatorial de correspondência múltipla foi utilizada para investigar a relação entre as variáveis do estudo (variáveis ativas) com o primeiro serviço de saúde procurado (variável passiva), o qual foi agrupado em:

Atenção básica: compreendem as UBS e USF, caracterizada pelo atendimento de demanda espontânea e programada, funcionamento em horário comercial, presença de equipe generalista e recursos de baixa densidade tecnológica;

UPA: caracterizada pelo atendimento de livre demanda, funcionamento 24 horas por dia, presença de equipe emergencialista e média densidade tecnológica;

Serviços especializados: compreendem os consultórios particulares, hospitais e ambulatórios de especialidades ou de referência, PCT e o SAE, presença de média e alta densidade tecnológica, equipes especializadas e apoio diagnóstico.

O projeto foi aprovado pelo Comitê de Ética da Faculdade de Medicina de São José do Rio Preto, conforme protocolo $7081 / 2008$.

\section{Resultados}

A principal porta de entrada para o diagnóstico da TB no município ao inicio dos sinais e sintomas foi a UPA $(49,4 \%)$. No entanto, o serviço de saúde que mais diagnosticou a doença foi hospital $(39,5 \%)$, seguido pelas unidades de atenção básica (30,9\%) (Tabela 1). Ressalta-se que a atenção básica diagnosticou 46,2\% dos doentes que a procuraram e a UPA apenas $20 \%$, e os demais diagnósticos dos usuários que procuraram pela UPA foram realizados nos hospitais $(42,5 \%)$ e na atenção básica (25\%).

A aplicação da análise fatorial privilegiou as dimensões 1 e 2 com valores próprios de 0,325 e 0,180 . A Tabela 2 indica a dimensão a que cada 
Tabela 1

Distribuição dos doentes com TB pulmonar segundo o primeiro serviço de saúde procurado e o local de diagnóstico da TB em São José do Rio Preto, São Paulo, Brasil, 2009

\begin{tabular}{|c|c|c|c|c|c|c|c|c|c|}
\hline \multirow{3}{*}{$\begin{array}{l}\text { Primeiro serviço } \\
\text { de saúde } \\
\text { procurado }\end{array}$} & \multicolumn{8}{|c|}{ Local de diagnóstico da tuberculose } & \multirow{3}{*}{$\begin{array}{l}\text { Total } \\
\text { n (\%) }\end{array}$} \\
\hline & \multirow{2}{*}{$\begin{array}{l}\text { UBS } \\
\text { n (\%) }\end{array}$} & \multirow{2}{*}{$\begin{array}{l}\text { USF } \\
\text { n (\%) }\end{array}$} & \multirow{2}{*}{$\begin{array}{c}\text { Ambulatório de } \\
\text { referência/Policlínicas } \\
\text { n (\%) }\end{array}$} & \multirow{2}{*}{$\begin{array}{l}\text { PCT } \\
\text { n (\%) }\end{array}$} & \multirow{2}{*}{$\begin{array}{l}\text { SAE } \\
n(\%)\end{array}$} & \multirow{2}{*}{$\begin{array}{l}\text { UPA } \\
\text { n (\%) }\end{array}$} & \multirow{2}{*}{$\begin{array}{c}\text { Consultório } \\
\text { particular } \\
\text { n (\%) }\end{array}$} & \multirow{2}{*}{$\begin{array}{l}\text { Hospital } \\
\text { n (\%) }\end{array}$} & \\
\hline & & & & & & & & & \\
\hline UBS & $11(45,8)$ & $2(8,3)$ & $1(4,2)$ & $2(8,3)$ & $1(4,2)$ & - & $1(4,2)$ & $6(25,0)$ & $24(100,0)$ \\
\hline USF & - & $1(50,0)$ & - & $1(50,0)$ & - & - & - & - & $2(100,0)$ \\
\hline $\begin{array}{l}\text { Ambulatório } \\
\text { de referência/ } \\
\text { Policlínicas }\end{array}$ & - & - & $1(50,0)$ & - & - & $1(50,0)$ & - & - & $2(100,0)$ \\
\hline PCT & - & - & - & $1(100,0)$ & - & - & - & - & $1(100,0)$ \\
\hline SAE & - & - & - & - & $2(100,0)$ & & & & $2(100,0)$ \\
\hline UPA & $7(17,5)$ & $3(7,5)$ & $2(5,0)$ & $2(5,0)$ & $1(2,5)$ & $8(20,0)$ & - & $17(42,5)$ & $40(100,0)$ \\
\hline $\begin{array}{l}\text { Consultório } \\
\text { particular }\end{array}$ & - & $1(33,3)$ & - & - & - & - & - & $2(66,7)$ & $3(100,0)$ \\
\hline Hospital & - & - & - & - & - & - & - & $7(100,0)$ & $7(100,0)$ \\
\hline Total & $18(22,2)$ & $7(8,6)$ & $4(4,9)$ & $6(7,4)$ & $4(4,9)$ & $9(11,1)$ & $1(1,2)$ & $32(39,5)$ & $81(100,0)$ \\
\hline
\end{tabular}

PCT: Programa de Controle da Tuberculose; SAE: Serviço de Atendimento Especializado; UBS: Unidade Básica de Saúde; UPA: Unidade de Pronto Atendimento; USF: Unidade Saúde da Família.

Tabela 2

Valores da contribuição absoluta $\left(\operatorname{Cos}^{2}\right)$ para as dimensões criadas.

\begin{tabular}{|c|c|c|c|c|}
\hline Variável (Legenda) & Categoria & $\begin{array}{c}\operatorname{Cos}^{2} \\
\text { Dimensão } 1\end{array}$ & $\begin{array}{c}\operatorname{Cos}^{2} \\
\text { Dimensão } 2\end{array}$ & Dimensão \\
\hline Procura pelo serviço de saúde mais próximo do domicílio & $\operatorname{Sim}(\mathrm{S})$ & 0,072731 & 0,168107 & 2 \\
\hline (SS mais próx.) & Não (N) & 0,072731 & 0,168107 & \\
\hline \multirow[t]{2}{*}{ Obtenção de consulta em 24 horas } & Consulta em 24 horas & 0,051175 & 0,431342 & 2 \\
\hline & Consulta $>24$ horas & 0,051175 & 0,431342 & \\
\hline \multirow[t]{3}{*}{ Hipótese diagnóstica (HD) } & Tuberculose (TB) & 0,528186 & 0,029978 & 1 \\
\hline & Não TB (outras doenças) & 0,230926 & 0,046958 & \\
\hline & Não informada (NI) & 0,050782 & 0,002355 & \\
\hline \multirow[t]{2}{*}{ Solicitação de baciloscopia (BK) } & $\operatorname{Sim}(S)$ & 0,698816 & 0,074922 & 1 \\
\hline & Não (N) & 0,698816 & 0,074922 & \\
\hline \multirow[t]{2}{*}{ Solicitação de raio-X (Rx) } & $\operatorname{Sim}(S)$ & 0,526708 & 0,219189 & 1 \\
\hline & Não (N) & 0,526708 & 0,219189 & \\
\hline Encaminhamento para consulta com outro profissional & $\operatorname{Sim}(\mathrm{S})$ & 0,059465 & 0,070533 & 2 \\
\hline (Encaminhamento) & Não (N) & 0,059465 & 0,070533 & \\
\hline Número de vezes que procurou os serviços de saúde até & Até 3 & 0,384450 & 0,296580 & 1 \\
\hline o diagnóstico & $>3$ & 0,384450 & 0,296580 & \\
\hline \multirow[t]{2}{*}{ Ocorrência de atraso no diagnóstico da TB } & Atraso & 0,239100 & 0,220134 & 1 \\
\hline & Não atraso & 0,239100 & 0,220134 & \\
\hline Diagnóstico realizado no primeiro serviço de saúde & $\operatorname{Sim}(S)$ & 0,343113 & 0,088676 & 1 \\
\hline procurado (Dx no $\left.1^{\circ} \mathrm{SS}\right)$ & Não (N) & 0,343113 & 0,088676 & \\
\hline
\end{tabular}


variável pertence, conforme a maior contribuição absoluta $\left(\operatorname{Cos}^{2}\right)$, e as variáveis que compõem a dimensão 1 explicam $29,2 \%$ da variabilidade dos dados. A dimensão 2 foi composta por variáveis que explicam $16,2 \%$ da variabilidade dos dados (Figura 1).

A atenção básica esteve associada à procura do doente pelo serviço de saúde mais próximo de seu domicilio, obtenção de consulta em mais de 24 horas, hipótese diagnóstica não informa$\mathrm{da}$, encaminhamento para consulta com outro profissional, mais de três visitas aos serviços de saúde e atraso para a obtenção do diagnóstico (Figura 1).
A UPA associou-se à não solicitação de raio-x e baciloscopia, hipótese diagnóstica de outras doenças além da TB e à não realização do diagnóstico nesse serviço (Figura 1).

Os serviços especializados tiveram associação com a não procura do doente pelo serviço de saúde mais próximo de seu domicílio, obtenção de consulta em até 24 horas, não encaminhamento para consulta com outro profissional, até três visitas aos serviços de saúde para a obtenção do diagnóstico e não atraso no diagnóstico (Figura 1).

A suspeição da TB, solicitação da baciloscopia de escarro e raio-x e realização do diagnósti-

Figura 1

Plano fatorial.

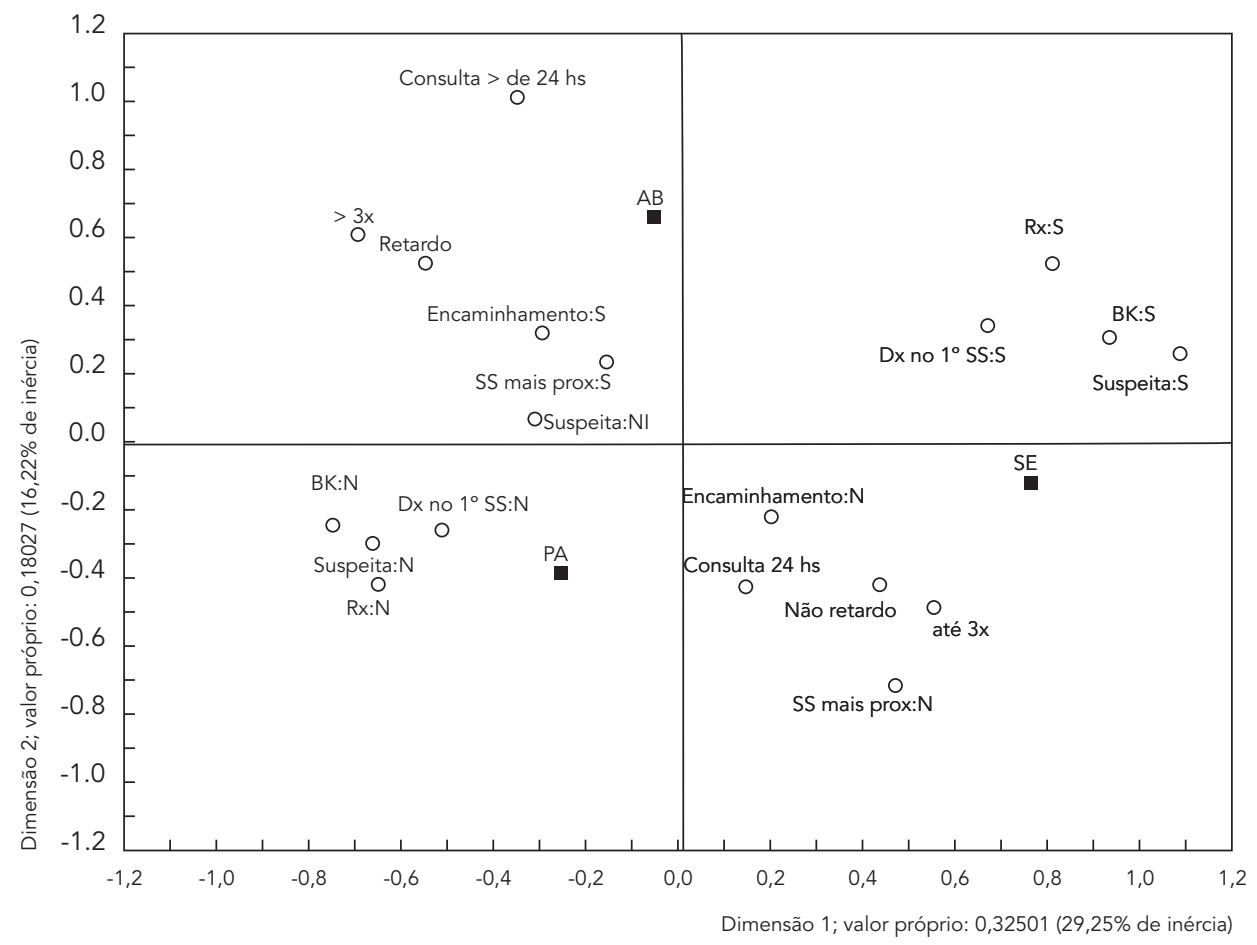

Serviços de saúde (AB: atenção básica; até 3x: procurou os serviços de saúde até 3 vezes para o diagnóstico; BK:N: não solicita baciloscopia; BK:S: solicita baciloscopia; Consulta 24hs: obtenção de consulta em até 24 horas; Consulta > 24hs: obtenção de consulta em mais 24 horas; Dx no 1ㅡ SS:N: diagnóstico não realizado no primeiro serviço de saúde procurado; Dx no 1ㅇ SS:S: diagnóstico realizado no primeiro serviço de saúde procurado; Encaminhamento:N: não encaminha para consulta com outro profissional; Encaminhamento:S: encaminha para consulta com outro profissional; Não retardo: não ocorrência de retardo entre os doentes; PA: Unidade de Pronto-atendimento; Retardo: ocorrência de retardo entre os doentes; Rx:N: não solicita raio-x; Rx:S: solicita raio-x; SE: serviço especializado; SS mais próx.:N: não procura pelo serviço de saúde mais próximo do domicílio; SS mais próx.:S: procura pelo serviço de saúde mais próximo do domicílio; Suspeita:N: suspeita diagnóstica de não tuberculose; Suspeita:NI: suspeita diagnóstica não informada pelo médico; Suspeita:S: suspeita diagnóstica de tuberculose; >3x: procurou os serviços de saúde mais de 3 vezes para o diagnóstico. 
co do primeiro serviço de saúde procurado não apresentaram associação com os três tipos de serviços analisados (Figura 1).

\section{Discussão}

Embora o município estudado apresente bons indicadores sociais, baixa incidência de TB e uma taxa de detecção de casos acima da meta pactuada pela OMS de $70 \%$, verifica-se ainda uma alta taxa de óbitos pela doença, o que pode estar relacionado a um baixo desempenho dos serviços de porta de entrada para o diagnóstico da doença, resultando em atraso na detecção de casos. Além disso, reflete o descompasso entre a organização da assistência da TB preconizada 3 , que define o controle da TB como responsabilidade da atenção básica, e a realidade encontrada, em que as UPA são a principal porta de entrada e os hospitais os locais que mais realizam o diagnóstico.

A literatura internacional aponta que a obtenção do diagnóstico da TB pode estar relacionada ao tipo de serviço de saúde procurado pelo usuário como porta de entrada 10,11,12,13. A procura pelas UPA no município estudado corrobora com a realidade brasileira de busca por serviços de saúde 25,26,27, sendo os possíveis motivos decorrentes de debilidades na ordenação do acesso aos serviços da rede assistencial, bem como da baixa resolubilidade e qualidade dos serviços de atenção básica, com horário de funcionamento e número de consultas restritas, aliadas à dificuldade de mudança nos hábitos culturais de procura por atendimento 26,27,28,29. A agilidade na obtenção de consulta, como mostrado neste estudo, também atrai os usuários às UPA para obter respostas mais rápidas às suas necessidades 26 .

A UPA, como uma das principais portas de entrada para o diagnóstico da TB, deveria estar qualificada para suspeitar e detectar os casos, bem como para realizar um trabalho conjunto/ articulado com os demais pontos de atenção no sentido de vincular o doente a sua unidade de procedência, possibilitando o seguimento e adequado manejo do caso. No entanto, neste estudo, a UPA apresentou o pior desempenho, e esteve associada à suspeição de outras doenças que não a TB, o que pode ter levado à não solicitação de baciloscopia de escarro e raio-x e, por consequência, à não realização do diagnóstico no primeiro serviço de saúde procurado.

Tais resultados refletem o despreparo dos profissionais para diagnosticar a TB, indo ao encontro da realidade do município, em que essas unidades (UPA) não são incluídas nos treinamentos e avaliações realizadas pelo PCT. Ade- mais, verifica-se que, na prática, a programação de treinamentos e a participação dos profissionais das UPA são dificultadas pela sobrecarga de trabalho e restrito número de recursos humanos, acarretando a centralização das informações em alguns profissionais que, com frequência, não as repassam ao restante da equipe.

Ainda no que se refere à qualificação profissional para o manejo da TB, é importante destacar a falta de sistematização nos treinamentos realizados pelas próprias equipes de atenção básica, ocorrendo somente treinamentos pontuais realizados pela equipe especializada do PCT. Nesse sentido, a construção de estratégias contextualizadas que promovam o diálogo entre as diretrizes políticas nacionais e a singularidade dos serviços de saúde ${ }^{5}$, com capacitações e supervisões contínuas dos profissionais de saúde, assumiria papel fundamental para o controle da TB no intuito de identificar falhas a serem enfrentadas na atenção/gestão e aprimorar habilidades 30 . Contudo, é de extrema importância que a proposta da educação permanente em saúde esteja inserida em uma rede de serviços organizada e articulada.

Portanto, o preparo do profissional para o controle da TB deveria se iniciar nas instituições de ensino técnico e superior, pela inclusão de guidelines com tempo suficiente para o ensino teórico e prático, a fim de capacitar o futuro profissional a lidar com a doença ${ }^{31}$. Entretanto, no Brasil, o ensino da TB na graduação ainda é insatisfatório, fragmentado e desvinculado dos programas de controle ${ }^{32}$, refletindo o modelo formador flexneriano.

Na análise da atenção básica como primeiro serviço de saúde procurado, faz-se necessário levar em consideração o grau de institucionalização da Estratégia Saúde da Família (ESF). Em 2009, o município encontrava-se no estágio de transição inicial da ESF, com cobertura de 14,2\% e pouca institucionalização no espaço da representação social e político, no qual o discurso e a agenda de prioridades estavam voltados à ampliação de UPA, reforçando a busca por atendimento nesses serviços. Nesse sentido, observa-se que as políticas de saúde do município não têm privilegiado ao longo dos anos a ampliação da cobertura da ESF. Ressalta-se que o fortalecimento da atenção básica enquanto porta de entrada e coordenadora da atenção requer a superação das políticas indutoras que competem entre si tanto pela expansão da atenção básica quanto pela construção de novas UPA.

Neste estudo, a proximidade dos serviços de atenção básica às residências dos usuários não necessariamente garantiu um melhor desempenho para o diagnóstico oportuno da TB (eviden- 
ciado pela associação com o atraso), haja vista a necessidade de o indivíduo ter de procurar mais de três vezes por serviços de saúde para ser diagnosticado, além de ter obtido consulta na atenção básica em mais de 24 horas.

Destaca-se que os serviços de atenção básica realizaram aproximadamente metade dos diagnósticos dos indivíduos que os procuraram, mas ainda existem desafios a serem enfrentados para melhorar ainda mais o desempenho da atenção básica no diagnóstico da TB.

Aspectos como a priorização das condições agudas, sobretudo as doenças sazonais, têm deixado as ações voltadas à busca de casos em segundo plano, sendo elas realizadas especialmente em época de campanha ou após capacitações. Como exemplo, nos últimos anos, houve um aumento de baciloscopias de escarro realizadas nas unidades de atenção básica, atingindo um ápice em 2008 (82,7\%), reflexo do maior investimento para a total desconcentração das ações de controle da doença (com capacitação e avaliações trimestrais das equipes de atenção básica); todavia, em 2009, o percentual de exames solicitados caiu para $57,5 \% 21$.

Outra questão relevante diz respeito à descontinuidade político-partidária ocorrida na última gestão do Município de São José do Rio Preto que resultou em um aumento na rotatividade dos profissionais de saúde, prejudicando a manutenção de equipes de saúde qualificadas para lidar com a TB.

Ainda na questão política, muitas vezes o espaço micro reproduz a definição e direcionamento do nível macro, que, no município, não priorizava a detecção de casos e sim os percentuais de cura da doença assumidos como compromisso no Pacto pela Vida 3 .

Mesmo que preconizados pelo Ministério da Saúde como métodos principais para a descoberta de casos de TB pulmonar, o raio-X e a baciloscopia de escarro foram pouco priorizados nos três tipos de serviços. A não priorização da baciloscopia, em especial, pode decorrer de falhas na sua operacionalização, desde a disponibilidade de insumos até a logística para o processamento do exame 33,34 , no entanto, em São José do Rio Preto, a não solicitação desses exames parece estar mais relacionada com a não suspeição da TB.

Apesar dos investimentos para desenvolver exames mais rápidos de diagnóstico da TB (entre eles, o GeneXpert-CEPHEID, Sunnyvale, Estados Unidos), o alcance de uma detecção eficaz de casos de TB, como evidenciado neste estudo, pode estar mais relacionadas à forma como os serviços de saúde se organizam para diagnosticar e tratar os casos de TB do que propriamente aos meios diagnósticos em si 12, o que reforça a necessidade de investimentos da gestão no componente processo, valorizando o reconhecimento do problema por intermédio da suspeita de TB.

Os serviços especializados, por mais que não sejam próximos ao domicilio dos doentes, apresentaram o melhor desempenho em relação ao diagnóstico da TB (obtenção de consulta em até 24 horas, sem encaminhamento para outros serviços, procura por serviços de saúde até três vezes e sem atraso no diagnóstico da TB), o que era esperado, visto que os serviços especializados em TB (PCT e SAE) possuem equipes preparadas e agilidade na oferta de testes de apoio diagnóstico. Frisa-se que todos os indivíduos que procuraram por esses serviços ao início dos sintomas da TB eram casos de recidiva, ou seja, já haviam tido contato prévio com o serviço e com a sintomatologia da doença. Dessa forma, optaram por buscar por atendimento em serviços que conhecidamente estavam aptos a realizar seu diagnóstico.

Neste estudo, encontrou-se o hospital como porta de entrada, o que não condiz com a forma de organização hierarquizada do sistema municipal de saúde, sendo a entrada nos hospitais públicos possível somente mediante encaminhamentos dos serviços de emergências (Pronto Atendimento ou Serviço de Atendimento Móvel de Urgência - SAMU) ou especializados. Entretanto, o fato de existirem hospitais privados cujos serviços de emergência são porta aberta para a população conveniada pode explicar esse dado.

Apesar de a TB ser uma condição sensível à atenção básica, neste estudo, um número significante de diagnósticos foi realizado em hospitais (39,5\%), o que pode ser decorrente da procura dos doentes pelos serviços de saúde em estágio avançado da doença 35,36, bem como deficiências na realização do diagnóstico oportuno da enfermidade em pauta pelos serviços de porta de entrada 37, resultando no encaminhamento dos usuários aos hospitais ainda sem diagnóstico. A descoberta dos casos de TB nos hospitais do município vem ocorrendo desde 2000, mesmo após a descentralização das ações de TB para a atenção básica e investimentos na estrutura dessas unidades 38 .

Frente ao exposto, os serviços de saúde que compõem a rede de atenção básica e as UPA não estão suficientemente preparados para a adequada detecção de casos de TB, sendo necessário determinar o fluxo dos usuários no sistema de saúde, fortalecendo os mecanismos de referência e contrarreferência, integrando os serviços que compõem a rede de atenção de modo que seja assegurada a continuidade da assistência e o acesso às ações e serviços de saúde nos diferentes níveis de atenção. 
A análise fatorial de correspondência múltipla privilegiou duas dimensões que, no conjunto, explicam $45,4 \%$ do desempenho dos serviços de porta de entrada para o diagnóstico da TB. Variáveis que envolvem aspectos da cultura organizacional dos serviços de saúde, bem como de formação e capacitação profissional, não foram possíveis de ser avaliadas pela perspectiva dos usuários, mas poderiam contribuir para a avaliação de tal desempenho. Neste sentido, enfatiza-se que, para a avaliação das ações em saúde, é necessária a percepção de todos os atores envolvidos no processo e a utilização de dados secundários.

Identificaram-se ainda como possíveis limitações deste estudo o viés de memória e o viés de informação, sobretudo quanto ao primeiro serviço de saúde procurado.

O alcance de uma detecção eficaz dos casos de TB nas principais portas de entrada perpassa pela melhoria na suspeição. A falta de suspeição da TB por parte dos profissionais de saúde encontrada neste estudo reflete o modo de organização do sistema de saúde do município, que apresenta limitações na capacitação em TB, restrita à atenção básica e prejudicada pela rotatividade dos recursos humanos.
Urge a necessidade de se pensar em estratégias que visem à qualificação e capacitação permanentes e sistematizadas dos profissionais de saúde que atuam nos diversos serviços que se constituem como portas de entrada da rede de atenção de modo a colaborar para a garantia dos conhecimentos/habilidades/competências dos profissionais no diagnóstico oportuno da TB. Além disso, os aspectos gerenciais do sistema de saúde do município e a descontinuidade político-administrativa não têm fortalecido a atenção básica como principal porta de entrada para o diagnóstico da doença, o que pode contribuir para a realização dos diagnósticos no âmbito hospitalar de maneira tardia.

Por fim, acredita-se que os resultados do estudo possam auxiliar na indução de um efetivo compromisso político que transcenda o marco fragmentado e reducionista do controle da doença nos diversos contextos sanitários, incluindo a detecção de casos de TB como meta prioritária no processo de pactuação vigente como elemento disparador da elaboração de planos locais compatíveis com o adequado controle da doença.

\section{Resumen}

Estudio transversal con el objetivo de analizar el desempeño del primer servicio de salud solicitado para el diagnóstico de la TB pulmonar en São José do Rio Preto, São Paulo, Brasil, en 2009. Se entrevistaron a 81 enfermos en tratamiento. Se adoptó un cuestionario basado en el Primary Care Assessment Tool, adaptado para atención a la TB, y el referencial de evaluación de los servicios de salud (estructura y proceso). Los datos se analizaron mediante técnicas descriptivas y análisis de correspondencia múltiple. El principal servicio solicitado fueron las urgencias (Pronto Atendimento-UPA) en un 49,4\% y el que más diagnosticó los casos de TB fue el hospital (39,5\%) y la atención primaria (30,9\%). Los servicios especializados presentaron una asociación con el mejor desempeño en el diagnóstico y la UPA con el peor. La atención básica se asoció con un desempeño intermedio y puntos débiles en la estructura. El alcance de una detección eficaz de los casos de TB, en las principales vías de acceso a los servicios sanitarios, pasa por la mejoría en la capacidad de inferencia de los síntomas, reforzando la necesidad de inversiones base en la capacitación de recursos humanos para la identificación de la sintomatología respiratoria.

Tuberculosis; Diagnóstico Tardío; Servicios de Salud; Atención Primaria de Salud 


\section{Colaboradores}

M. A. Z. Ponce participou da concepção do projeto, análise e interpretação dos dados e redação do artigo. A. Ruffino-Netto, A. A. Monroe, S. H. F. Vendramini, T. Arakawa, R. L. P. Andrade, B. E. Scatolin e A. D. Wysocki participaram da redação do artigo e revisão crítica relevante do conteúdo intelectual. L. M. Scatena participou da análise e interpretação dos dados e redação do artigo. T. C. S. Villa participou da concepção do projeto, análise e interpretação dos dados e revisão crítica relevante do conteúdo intelectual.

\section{Referências}

1. Starfield B. Atenção primária: equilíbrio entre necessidades de saúde, serviços e tecnologia. Brasília: Ministério da Saúde/Fundo das Nações Unidas para a Infância; 2002.

2. Mendes EV. As redes de atenção à saúde. Belo Horizonte: Escola de Saúde Pública do Estado de Minas Gerais; 2009.

3. Coordenação de Apoio à Gestão Descentralizada, Secretaria-Executiva, Ministério da Saúde. Diretrizes operacionais para os pactos pela vida, em defesa do SUS e de gestão. Brasília: Ministério da Saúde; 2006.

4. Mendes EV. As redes de atenção à saúde. Ciênc Saúde Coletiva 2010; 15:2297-305.

5. Ministério da Saúde. Portaria MS/GM no 2.488, 21 outubro 2011. Aprova a Política Nacional de Atenção Básica, estabelecendo a revisão de diretrizes e normas para a organização da Atenção Básica, para a Estratégia Saúde da Família (ESF) e o Programa de Agentes Comunitários de Saúde (PACS). Diário Oficial da União 2011; 24 out.

6. Ministério da Saúde. Decreto no 7.508 de 28 de julho de 2011. Regulamenta a Lei no 8.080 de 19 de setembro de 1990, para dispor sobre a organização do Sistema Único de Saúde - SUS, o planejamento da saúde, a assistência à saúde e a articulação interfederativa, e dá outras providências. Diário Oficial da União 2011; 29 jun.

7. Machado K. Novidades no SUS. Radis Comunicação em Saúde 2009; (83):12-6.

\section{Agradecimentos}

Ao CNPq pelo financiamento. A FAPESP pela bolsa de doutorado direto (processo 2009/51684-1). (Edital MCT/CNPq/CT-Saúde/Ministério da Saúde/SCTIE/ DECIT 67/2009 - processo 558835/2009-0).
8. Mendes EV. Atenção primária à saúde no SUS. Fortaleza: Escola de Saúde Pública do Ceará; 2002.

9. Organização Mundial da Saúde. Cuidados inovadores para condições crônicas: componentes estruturais de ação: relatório mundial. Brasília: Organização Mundial da Saúde; 2003.

10. Diéz M, Bleda MJ, Alcaide J, Caloto T, Castells C, Cardenal JI, et al. Determinants of patient delay among tuberculosis cases in Spain. Eur J Public Health 2004; 14:151-5.

11. Santos MAPS, Albuquerque MFPM, Ximenes RAA, Lucena-Silva NLCL, Braga C, Campelo ARL, et al. Risk factors for treatment delay in pulmonary tuberculosis in Recife, Brazil. BMC Public Health 2005; 5:25.

12. Leung ECC, Leung CC, Tam CM. Delayed presentation and treatment of newly diagnosed pulmonary tuberculosis patients in Hong Kong. Hong Kong Med J 2007; 13:221-7.

13. Wang W, Jiang Q, Abdullah ASM, Xu B. Barriers in accessing to tuberculosis care among non-residents in Shanghai: a descriptive study of delay in diagnosis. Eur J Public Health 2007; 17:419-23.

14. World Health Organization. Global tuberculosis control: surveillance, planning, financing: $\mathrm{WHO}$ report. Geneva: World Health Organization; 1999.

15. Ignotti E, Oliveira BFA, Hartwig S, Oliveira HC, Scatena JHG. Analysis of the Tuberculosis Control Program in the city of Cáceres, Brazil, prior to and after the implementation of a Family Health Program. J Bras Pneumol 2007; 33:287-94. 
16. Varma JK, Wiriyakitjar D, Nateniyom S, Anuwatnonthakate A, Monkongdee P, Sumnapan S, et al. Evaluating the potential impact of the new Global Plan to Stop TB: Thailand, 2004-2005. Bull World Health Organ 2007; 85:586-92.

17. Gómez PRM, Achiong FE, Rigau JMM, Núñez LV, Hernández JQ, Fundora CGP. Evaluación de los indicadores operacionales del programa de la tuberculosis. Matanzas 2008; 30(5). http://www. revmatanzas.sld.cu/revista\%20médica/ano\%20 2008/vol5\%202008/tema7.htm (acessado em 10/ Ago/2009).

18. Soltan V, Henry AK, Crudu V, Zatusevski I. Increasing tuberculosis case detection: lessons from the Republic of Moldova. Bull World Health Organ 2008; 86:71-6.

19. Kruk ME, Prescott MR, Pinho H, Gálea S. Are doctors and nurses associated with coverage of essential health services in developing countries? A cross-sectional study. Hum Resour Health 2009; $7: 27$

20. Scatena LM, Villa TCS, Ruffino Netto A, Kritski AL, Figueiredo TMRM, Vendramini SHF, et al. Dificuldades de acesso a serviços de saúde para diagnóstico de tuberculose em municípios do Brasil. Rev Saúde Pública 2009; 43:389-97.

21. Secretaria Municipal de Saúde e Higiene de São José do Rio Preto. Controle de tuberculose ARE/ VE. Situação da tuberculose em São José do Rio Preto. São José do Rio Preto: Secretaria Municipal de Saúde e Higiene de São José do Rio Preto; 2009.

22. Almeida C, Macinko J. Validação de uma metodologia de avaliação rápida das características organizacionais e do desempenho dos serviços de atenção básica do Sistema Único de Saúde (SUS) em nível local. Brasília: Organização Pan-Americana da Saúde; 2006.

23. Villa TCS, Ruffino-Netto A. Questionário para avaliação de desempenho de serviços de atenção básica no controle da tuberculose no Brasil. J Bras Pneumol 2009; 35:610-2.

24. Hurst J, Jee-Hughes M. Performance measurement and performance management in OECD health systems. Paris: Organisation for Economic Cooperation and Development; 2001. (OECD Labour Market and Social Policy Occasional Papers, 47)

25. Cecilio LCO. Modelos tecno-assistenciais em saú de: da pirâmide ao círculo, uma possibilidade a ser explorada. Cad Saúde Pública 1997; 13:469-78.

26. Marques GQ, Lima MAD. Demandas de usuários a um serviço de pronto atendimento e seu acolhimento ao sistema de saúde. Rev Latinoam Enferm 2007; 15:13-9.

27. Oliveira LH, Mattos RA, Souza AIS. Cidadãos peregrinos: os "usuários" do SUS e os significados de sua demanda a prontos-socorros e hospitais no contexto de um processo de reorientação do modelo assistencial. Ciênc Saúde Coletiva 2009; 14:1929-38.
28. Santos JS, Scarpelini S, Brasileiro SLL, Ferraz CA, Dallora MELV, Sá MFP. Avaliação do modelo de organização da Unidade de Emergência do HCFMRP-USP, adotando, como referência, as políticas nacionais de atenção às urgências e de humanização. Medicina Ribeirão Preto 2003; 36: 498-515.

29. Zerbini E, Chirico MC, Salvadores B, Amigot B, Estrada S, Algorry G. Delay in tuberculosis diagnosis and treatment in four provinces of Argentina. Int J Tuberc Lung Dis 2008; 12:63-8.

30. Monroe AA, Gonzalez RIC, Palha PF, Sassaki CM, Ruffino-Netto A, Vendramini SHF, et al. Envolvimento de equipes da Atenção Básica à Saúde no controle da tuberculose. Rev Esc Enferm USP 2008; 42:262-7.

31. Caminero JA. Is the DOTS strategy sufficient to achieve tuberculosis control in low- and middleincome countries? Need for interventions in universities and medical schools. Int J Tuberc Lung Dis 2003; 7:509-15.

32. Villa TCS, Firmino DR, Andrade RLP, Brunello MEF, Oliveira MF, Ruffino-Netto A. Practical tuberculosis teaching in nursing schools: a survey. Online Braz J Nurs (Online) 2006; 5(3). http://www.objnursing. uff.br/index.php/nursing/article/view/608.

33. Nogueira JA, Ruffino Netto A, Monroe AA, Cardozo Gonzales RI, Villa TCS. Busca ativa de sintomáticos respiratórios no controle da tuberculose na percepção do Agente Comunitário de Saúde. Rev Eletrônica Enferm 2007; 9:106-18.

34. Marcolino ABL, Nogueira JN, Ruffino-Netto A, Moraes RM, Sá LD, Villa TCS. Avaliação do acesso às ações de controle da tuberculose no contexto das equipes de saúde da família de Bayeux - PB. Rev Bras Epidemiol 2009; 12:144-57.

35. Yimer S, Bjune G, Alene G. Diagnostic and treatment delay among pulmonary tuberculosis patients in Ethiopia: a cross sectional study. BMC Infect Dis 2005; 5:112.

36. Sengupta S, Pungrassami P, Balthip Q, Strauss R, Kasetjaroen Y, Chongsuvivatwong V, et al. Social impact of tuberculosis in southern Thailand: views from patients, care providers and the community. Int J Tuberc Lung Dis 2006; 10:1008-12.

37. Arcêncio RA, Oliveira MF, Villa TCS. Internações por tuberculose pulmonar no Estado de São Paulo no ano de 2004. Ciênc Saúde Coletiva 2007; 12: 409-17.

38. Santos MLSG, Villa TCS, Vendramini SHF, Cardozo Gonzales RI, Palha PF, Santos NSGM, et al. A gerência das ações de controle da tuberculose em municípios prioritários do interior paulista. Texto \& Contexto Enferm 2010; 19:64-9.

Recebido em 28/Ago/2012

Versão final reapresentada em 18/Dez/2012 Aprovado em 08/Jan/2013 